\title{
Psychiatric Stigma in Developing Societies
}

\section{Saeed Shoja Shafti}

Full Professor of Psychiatry, University of Social Welfare and Rehabilitation Sciences [USWR], Razi Psychiatric Hospital, Tehran - Iran

*Corresponding Author: Saeed Shoja Shafti, Full Professor of Psychiatry, University of Social Welfare and Rehabilitation Sciences [USWR], Razi Psychiatric Hospital, Tehran - Iran

Received date: January 09, 2021; Accepted date: March 01, 2021; Published date: March 05, 2021

Citation: Saeed S Shafti. (2021) Psychiatric Stigma in Developing Societies. Biomedical Research and Clinical Reviews. 3(3);

DOI: $10.31579 / 2692-9406 / 042$

Copyright: (c) 2021 Saeed Shoja Shafti, This is an open-access article distributed under the terms of the Creative Commons Attribution License, which permits unrestricted use, distribution, and reproduction in any medium, provided the original author and source are credited.

\begin{abstract}
:
Stigmatizing attitudes toward persons with mental syndromes are prevalent in the general population and even among mental health professionals, a problem that may result easily in public avoidance, constant discrimination, and declined help-seeking behavior. The effect of stigma is twofold: Public stigma is the response that the public has to people with mental disorder. Self-stigma is the bigotry which persons with mental disorder turn against themselves. The WHO has advised that stigma is one of the largest barricades to treatment engagement, even if management is operative, even in low-income nations. While before and according to a series of researches the outcome of severe mental illness is generally better in developing societies than in developed countries, and it has been suggested that stigma is less severe or non-existent in unindustrialized nations, the current studies and observations do not confirm such an optimistic hint and the idea that stigma attached to mental illness is a global phenomenon seems a reasonable inference. In the present article, the issue of stigmatization, deinstitutionalization, national goal setting, and real situation of various modules of psychiatric rehabilitation, in the context of social or public psychiatry, especially in developing countries, is discussed, from a practical point of view.
\end{abstract}

Key words: chronic mental patient; severe mental illness; psychiatric rehabilitation; public psychiatry; social psychiatry; stigma

\section{Introduction:}

A mental disorder is a "clinically significant behavioral or psychological disorder that occurs in a person and that is associated with present distress or disability or with a meaningfully increased risk of suffering death, pain, disability, or an important loss of freedom," which results from "a manifestation of a behavioral, psychological, or biological dysfunction in the individual" [1]. Stigmatizing attitudes toward persons with mental syndromes are prevalent in the population and even among mental health experts. Unlike people with physical disabilities, those with mental conditions are often observed by the public to be in control of their debilities and accountable for causing them [2]. The effect of stigma is twofold: Public stigma is the response that the general public has to individuals with mental disorder. Self-stigma is the bigotry which persons with mental disorder turn against themselves. Both public and self-stigma may be assumed in terms of three elements: stereotypes, prejudice, and discrimination [3]. The WHO has advised that stigma is one of the largest barricades to treatment engagement, even if management is operative, even in low-income nations [4]. Stigmatization of people with mental disorders may result in public evading, steady discrimination, and declined help-seeking behavior. In a survey in north America, more than one half of the respondents reported that they were reluctant to spend an evening socializing with, work next to, or have a family member marry a person with mental illness. Shame is reported as one of the main obstacles from seeking help for mental disorders in both developing and developed societies [5]. As a result, stigma is as a possible key reason in mental health services access and use, particularly underutilization of obtainable facilities by some segments of society, most markedly minority racial/ethnic groups [6]. Goffman believed that stigma is an actual/inferred attribute that damages the bearer's reputation, and degrades him/her to a socially discredited situation. Hence, social devaluation and rejection are customary experiences of the stigmatized. Affiliation with the stigmatized, as well, causes a secondary stigma courtesy stigma [7]. Nonetheless, modified labeling theory, recognizes the sociocultural context of stigma, a social construct that reflects relations of power operating at societal levels [8]. Within modified labeling theory, powerful groups in society impose stereotypically negative labels on those they suppose unwelcome, whom they later devalue and discriminate [9]. This conceptualization of stigma corresponds with a social psychology standpoint that stigmatization links with human cognition via stereotyping and prejudices [10]. For instance, some studies have detected that clinicians exhibit 'unintentional biases' in their decision and responses to patients and their relatives, in spite of sincere commitments to providing patient-centered and culturally sensitive care [11]. Self-stigma theory further postulates that some, among the socially devalued and discriminated patients, internalize public stigma by underestimating themselves and unsuitably changing their behavior and approaches [12]. The institutional context for the stigmatization of consumer has also been explored in a number of studies. Most institutional stigma research has focused on service providers, i.e., psychiatric and primary physicians, psychologists, other healthcare professionals - as stigmatizers, rather than the institutions themselves as stigmatizers [13]. Despite the high prevalence of these conditions, recognized managements have revealed effectiveness in modifying the problem and improving personal functioning in society [14]. Building from Goffman's initial conceptualization [15], Jones and colleagues [16], 
also, acknowledged six dimensions of stigma. These include concealability, course, disruptiveness, peril, origin, and aesthetics [17]. Moreover, Corrigan and other scholars [18, 19] recognized dimensions of stability, controllability, and pity. It is important to understand that these dimensions can either present independently or simultaneously to create stigma [17].

\section{Background:}

Far more than any other type of disease, mental syndromes are subject to negative verdicts and stigmatization [20]. Many patients not only have to cope with the often devastating effects of their disorder, but also suffer from social rejection and biases. Stigmatization of the mentally ill has a long tradition, and the word "stigmatization" itself indicates the negative connotations: in ancient Greece, a "stigma" was a brand to mark slaves or criminals [21]. For centuries, society did not treat persons suffering from depression, autism, schizophrenia and other mental illnesses better than slaves or offenders: they were imprisoned, tortured or executed. During the Middle Ages, mental disorder was considered as a punishment from God: sufferers were believed to be haunted by the evil spirit and were burned at the stake, or thrown in prison and madhouse where they were chained to the walls or their beds. During the Enlightenment, the mentally ill patients were finally freed from their chains and institutions were established to help sufferers of mental disorder [21]. Stigmatization of mental disorder is still a main social problem. The common people are mostly ignorant about this problem, and fear of the mentally ill remains ubiquitous. Structural discrimination of the mentally ill is still pervasive, whether in legislature or in recovery efforts. Stigma can be defined on three conceptual levels: cognitive, emotional and behavioral, which allows us to separate mere stereotypes from preconception and discrimination. Stereotypes refer to manufactured beliefs and approaches towards members of certain groups, such as ethnic or religious groups, as well as the mentally ill. The most prominent stereotypes surrounding the mentally ill suppose unpredictability, unreliability and dangerousness; patients with schizophrenia are most affected by such outlooks [21]. A scientific concept on the stigma of mental disorder was first developed in the middle of the $20^{\text {th }}$ century, first hypothetically and finally empirically in the 1970s. The book Stigma: Notes on the Management of Spoiled Identity, published in 1963 by the American sociologist Erwin Goffman, laid the foundation for stigma study as a scientific discipline and described how stigmatized persons deal with the challenge [21]. Goffman was very critical of mental hospitals because these further increased stigmatization instead of enabling patients to lead normal lives. This was in line with many of his contemporary scientists, including Scheff, Thomas Szasz, Ronald Laing and Michel Foucault, who claimed that the stigmatizing consequences of mental disorder could be attributed to how psychiatry was structured and not to the mental disease itself [21]. The stigma attached to mental illness is omnipresent. There is no country, society or culture where people with mental disorder have the same shared value as people without a mental sickness [22]. While stigma is widespread, the experience of the stigmatized individual is influenced by culture. For example, the role of supernatural, religious or magical explanations of mental disorder still succeeds in many non-Western societies. There are also differences in stigmatization depending on the type of ailment [23, 24, and 25]. In a Swedish study on relatives of persons with mental disorders, a substantial number indicated that the ill relative would be better off dead and/or wished that the patient and the relative had never met or that the patient had never been born [26]. On the other hand, offering organic reasons for these maladies might have detrimental effects in terms of acceptance and integration of the mentally ill [21]. Up till now, stigma toward persons with mental disorder is a severe social problem, in addition to a heavy burden for affected people [27, 28]. Indeed, though the scientific literature showed that people usually perceive individuals with mental disorder as dangerous [29], scientific literature have demonstrated that the a significant relationship between the majority of psychiatric disorders and violent behaviors does not exist [30], and therefore attitudes toward people with mental illness are in most cases related to a biased prejudice and/or stereotype [31, 32]. Since the cause to which the mental illness condition is attributed [psycho-sociological vs. biogenetic] has been considered to be one of the main factors underlying the stigmatizing processes [17], etiological beliefs have been used as a promotional trigger to overcome stigma in several public health programs intended to reduce discrimination toward people with mental disorder. Accordingly, mental disorders should be regarded as medical conditions that should be treated with medical treatments, as like as all other ailments [33, 34 and 35].

\section{Contemporary DE-Stigmatization Strategies:}

Plans for addressing stigmatization of persons with mental syndromes can be divided into three groups: Protest, education, and contact. There are some proofs that protest against prejudiced descriptions of mental illness may be effective in decreasing stigmatizing conducts against individuals with mental illnesses. Information/education about mental illness may promote a better understanding of mental disorder, and educated people are less probable to support stigma and discrimination. Also, an inverse association between having contact with a person with mental disorder and endorsing stigmatizing manners has been documented [5]. Three "channels", too, are used to mediate these policies: mass media, opinion leaders and persons of trust [21]. On the other hand, family therapy, as well, may help relatives understand psychiatric conditions and how they can help/support the distressed person [36]. Some research recommends that more care to relatives of persons with mental illness is required [37]. Besides addressing public opinions of mental ailment, psychiatrists, also, need to look at their own exercise because the task of the psychiatrist is much more than making a diagnosis and providing an appropriate label. People with mental disorder need help in making sense of their capabilities. As in other divisions of medicine, suitable and acceptable managements need to be built on the grounds of good interactions in which patients feel heard and understood. Empathy and support are expected to increase a positive relationship while retributive actions and insensible approaches will do the opposite [38, 39]. Anti-stigma interventions should normalize the experience of mental disorder and target perceptions that persons with mental disorder are dangerous. Such interventions can be integrated into schoolbased curriculum and target social groups [e.g., those with fewer years of education] that constantly endorse negative opinions regarding people with mental disorder [40]. Furthermore, it should focus on increasing positive personal contact with patients with mental disorder, target influential persons [e.g., landlords, employers], and should incorporate messages about the ways in which stigma and discrimination hinders life objectives and chances [37]. Besides, interventions should be tailored to the concerns, assets, and social position of a well-defined milieu or group. Such tailoring can augment the relevancy and efficiency of the intervention. Additionally, many, unceasing contacts with persons with mental disorder is encouraged as multiple positive contacts more successfully diminish stigma than a single meeting [41]. Also, fear of the stigmatizing effects of management has declined by providing care in less stigmatizing surroundings like primary care offices, community centers, and schools [42]. On the other hand, while anti-stigma interventions was on the hypothesis that increased awareness and acknowledgment of mental disorder will lead to decreases in stigma, increased mental health knowledge has been related sometimes with untouched or even increased levels of stigma [43].

\section{Discussion:}

Stigmatization in psychiatry, while is abstractly similar to stigmatization due to chronic contagious diseases, like leprosy or tuberculosis, is 
practically different from it. It involves loss of function, loss of safety, and loss of ordinary social relations, which are not easily responsive to current drug treatments or standard therapeutic strategies. For example, while in leprosy, keeping social distance and treatment of the disease with available drugs can improve the personal function and feeling of safety in others in short-term, in schizophrenia, neither of the said deficiencies are retrievable acceptably even in long-term. On the other hand, there are some differences between stigmatization in the developed societies and developing countries. For example while stigmatization in developed societies can be established more rapidly or remarking, in developing or non-developed nations it may develop later or more blunted. But finally and in any case it will develop. No tribe or nation in the world can accept the erratic behavior of its fellow citizen, who has lost his or her insight regarding the actualities of real world and has turned into a burdensome individual that demands persistent unidirectional free care, food and shelter. In addition to said requirements, his or her dangerous behavior, as well, should be considered, which may jeopardize insistently the patient's or relatives' life and wellbeing. On the other hand, occasional or likely abusing of psychiatric labeling by some of mental patients for deliberate performance of crime, increase the subjective sense of unpredictability in this regard. Though many of positive symptoms or crazy conducts may decrease by means of reasonable treatments, the remaining deficit syndrome in schizophrenia, partial remission or recurrent relapses in mood disorders, drug non-compliance or treatment-refractoriness in both, suicidal or homicidal threats, uncontrollable aggression and irritability, inability to work or insecure employment, and failure to respond appropriately to financial or monetary necessities, can reinforce the stigmatization so inflexibly that no preaching or lecture can overcome that. Here, one of the major differences between developed societies and developing nations is traditional tolerance of misfortunes, which seems to be more inevitable in the later ones. After invention of psychotropic drugs, especially chlorpromazine in the early1950s, establishment of community psychiatry with its wide-ranging goals, or better to say, rehabilitation of chronic mental patients, became conceivable. So, deinstitutionalization and discharge of severe and chronic mental patients in short-term constituted the main purpose of modern public psychiatry. Facilities, like community mental health centers, with their multidisciplinary services, and related agenda, like establishing case management network, residential management, crisis intervention services, vocational rehabilitation, self-help groups or psychosocial clubs, assertive outreach, day hospitals, night hospitals, and associated psychosocial interventions, like behavioral family management, altogether intended to improve chronic mental patient's function and sociability, parallel to decline his or her vulnerability by pharmacotherapy. But such a philanthropic program could not be accomplished on a national scale without its own specific budget, staff, and settings. Also, it needs to be followed or executed incessantly, habitually and in all places, not scholastically or occasionally. Without such preparations, deinstitutionalization has no result except than upsurge of wandering, homelessness, delinquency, addiction, and familial chaos, which can be accounted as real threats against normal and functional part of society. Surely, basic and citizens' rights, as well, advocate functional and vocational rehabilitation of chronic mental patients up to a level approximately similar to pre-morbid situation, and turning helpless, unstable and unemployed patients into independent, working and reliable persons. But such compassionate goals, in short-term and long-term, are not reachable by incomplete maneuvers or facilities. While such a dilemma is not restricted to undeveloped nations, and is observable relatively in technologically advanced societies too, its shortage is more palpable in the unindustrialized civilizations. Even now, tactics like case management, behavioral family management, psycho-education, which does not demand specific financial plan or amenities, except than devoted labor force, do not have a full and distinct role in rehabilitative packages, and above all, still the concepts of rehabilitation and independency have not been well-defined nationally and practically. Case management, which is a vital opening factor in community psychiatry, has not found its prearrangement and enthusiasts, whether pedagogically or executively, and has yet an unsettled role. Social skills training, whether in the frame of basic training, or modules or attention - focusing procedure, for selfmanagement of areas like medication, home finding and maintenance, recreation and leisure skills, budget management, usage of public agencies, training and enhancement of interactional, communicative and conversational skills, in family and social areas, and finally problem solving skills, has not been considered systematically as a core part of preliminary psychosocial rehabilitation. Crisis intervention services, like telephone support, home support, community home support or crisis lodging, in many places or occasions, does not have a schematic plan. Housing of chronic psychiatric patients after release from hospital, which is a very important part of the rehabilitation, and consists of locations like half-way houses, group houses, supervised or satellite apartments are not accessible, even in large cities or urban areas. Vocational rehabilitation, with its basic elements like work skills assessment, work adjustment, job skills training, sheltered employment, transitional employment, job finding and job maintenance, which is a key element designed for making self-governing and working people, is usually doable partially and recreationally, not completely and enthusiastically [44]. Why the situation is so after several decades of official commencement of psychiatric rehabilitation in developed societies? Why in many of the developing countries it is still in the pilot phase of assessment, or maybe in complete ignorance? Why deinstitutionalization program has not been successful enough, in spite of evolution of psychotropic medications? Two studies of WHO have found that the outcome of schizophrenia was generally better in developing societies than in developed countries, which was associated by the setting; acute onset; being married or cohabiting with a partner; and having access to a supportive network [45]. Similar findings about better outcomes of schizophrenia in developing countries were found by other investigators, too [46, 47]. So, while it is suggested that stigma may be less severe in developing countries [4549], a study carried out in India, in which 463 persons with schizophrenia and 651 family members were interviewed in four cities, reported that two thirds of the respondents had suffered discrimination [5]. In Ethiopia, also, among relatives of people with a diagnosis of schizophrenia or mood disorders, $75 \%$ reported that they had experienced stigma due to the presence of mental illness in the family, and $37 \%$ wanted to conceal the fact that a relative was ill [5]. In a large survey carried out in China, too, among people with schizophrenia and their relatives, $50 \%$ of the respondents reported they had felt significantly stigmatized [5]. Also, in a similar study in Iran, $45 \%$ of relatives of people with diagnosis of schizophrenia and $32.5 \%$ of relatives of depressive patients had experienced discrimination and humiliation and suffering from the stigmatization, which in turn had made them feel uncomfortable and ashamed [50]. So while the above proofs are not in harmony with the verdicts of Jablensky [46], Hopper et al. [47], Fabrega [48] and $\mathrm{Ng}$ [49], they challenge the conclusion of Dols, too, who believed that there is almost no stigmatization in Islamic societies [51]. So, the idea that stigma attached to mental illness is a global phenomenon seems a reasonable inference [22]. Also, disregard to probable neglects or faults, since the "disturbance of function" due to clinical signs and symptoms constitutes the basis of psychiatric disorders and diagnoses in 'Diagnostic and statistical manual of mental disorders [DSM]' and 'International Statistical Classification of Diseases and Related Health Problems [ICD]', an actual fact which is always noticeable in clinical or social settings, critical standpoints of some of scholars regarding the likely role of psychiatric organizations and services in generation of stigmatization [21] do not seem to be an exact or working extrapolation. Higher acceptance of psychiatric patients by associated relatives in developing societies, in comparison with the developed nations, does not 
signify heartfelt compliance of families towards wrongdoings of other members who suffer from severe mental illness, or guaranteeing patient's independency and good fortune. It is more comparable to the fact that misdemeanors of a crazy person have more echoes in complicated urban zones than in isolated rural regions. Families in developing societies usually tolerate or keep their ill relatives because, culturally, they see no alternative except than that. They cannot select other plans because their kindred and cultural values expect them to comply stereotypically with the existent scheme, even if it has detrimental outcomes. On the other hand, no national program can be achieved successfully when no social necessity or core insight demands that. Moreover, it is noticeable that incongruity between program, staff, setting and culture can undermine the end-result of every blueprint, disregard to its usefulness or principles. Then again, neoliberalism and privatization, as newfangled global economical policies, with their profit-seeking and progresspursuing objectives, have challenged, intellectually and fiscally, the psychiatric rehabilitation, with its basically liberal purposes, which perceives economical independency of mental patient a primary objective and appreciates one-sided spending of money for the sake of rehabilitation a right, not charity. So, there is a basic discrepancy between the said outlooks. Consequently, with respect to psychiatric rehabilitation, the resultant side effects of such a process in developing countries with more vulnerable economic foundations can be more confusing than advanced societies with more stable financial competencies. Also, integrating psychiatric wards into general hospitals, along with its unavoidable side effects and restrictions, reduction of public psychiatric beds and holding chronic mental patients in private sheltered sanatoriums, and turning psychiatric units into medical treatment centers, are generally in conflict with the philosophy of social psychiatry and deactivate strategic purposes of psychiatric rehabilitation. Such movements show that the deinstitutionalization has not been in cooperation with the psychiatric rehabilitation and has only been disguised according to the social necessities and circumstances. So, the roots of stigmatization in psychiatry, which has been stated before, have not been faded methodically and by way of rehabilitation in the last decades, and therefore expecting improvement of stigmatization could not be a faithful anticipation. Though psychotropic medications have paved the way for great optimisms, their attainment is not imaginable without systematic endeavors, investments and policy adjustments. Improving managerial rehearses by training of enthusiast apprentices in fellowship of public psychiatry, clarification of associated programs for political and administrative decision makers, for planning of necessary finances and beginning of multidisciplinary approaches, formulating necessary packages for measured amendment of cultural aspects of stigma, are among the key elements for solving the present problem. If peoples' distrust is due to patients' misbehavior, whether intentionally or unintentionally, then it is not correctable without acceptable behavioral recovery. In conclusion, considering the abovementioned background and outcomes of methodical studies, it seems that assessing stigma is a very complex issue. Indeed, it involves complex aspects, such as etiological beliefs, attitudes, prejudices, personal, and social problems, both toward mentally ill persons and in the mental disorders' perceiver, while taking into account the role of different cultures. Moreover, and of fundamental significance in this context is the distinction of different mental disorders, such as schizophrenia, depression, and addiction, which should be studied individually to highlight the specificity of their relations with stigma [52].

\section{Conclusion:}

Due to a series of cultural, financial and administrative reasons, deinstitutionalization and psychiatric rehabilitation have not been taken place meticulously in developing countries in the last decades, and in consequence improvement of stigma has never been a conceivable or achievable goal. Achievement of such a valuable objective at a national level, demands a sequences of social, fiscal and executive amendments, which may not, as well, be an easy task in the current unstable situation of world. Nullifying the "disturbance of function" by means of psychopharmacotherapy and thorough psychosocial rehabilitation is the main key for modification of stigma.

\section{References}

1. Washington, D.C.American Psychiatric Association. (2000) Diagnostic and statistical manual of mental disorders [4th Ed., Text Revision].

2. Douglas M. (1994) The construction of the physician: a cultural approach to medical fashions. In: Budd S, Sharma U, eds. The healing bond: the patient-practitioner relationship and therapeutic responsibility. London: Routledge.

3. Britten N. (1994) Patients' ideas about medicines: a qualitative study in a general practice population. Br J Gen Pract. 44:465-468.

4. Patel V, Araya R, Chatterjee S, Chisholm D, Cohen A, De Silva M, et al. (2007) Treatment and prevention of mental disorders in low-income and middle-income countries. Lancet. 370 [9591]: 991-1005.

5. Maj M. (2009) World Aspects of Psychiatry: Stigmatizing Attitudes Toward People With Mental Disorders. In: Sadock BJ, Sadock VA, Ruiz P, eds. Kaplan \& Sadock's Comprehensive Textbook of Psychiatry. 9th edition. Vol.1I. Philadelphia: Lippincott Williams \& Wilkins, 4516.

6. Mukolo A, Heflinger CA, Wallston KA. (2010) The stigma of childhood mental disorders: A conceptual framework. J Am Acad Child Adolesc Psychiatry. 49[2]: 182-198.

7. Goffman, E. (1963) Stigma: Notes on the management of spoiled identity. Englewood Cliffs, NJ: Prentice Hall.

8. Link BG, Phelan JC. (2001) Conceptualizing stigma. Annual Review of Sociology. 27: 363-385.

9. Link BG, Cullen FT, Struening EL, Shrout PE, Dohrenwend BP. (1989) A Modified labeling theory approach to mental disorders: an empirical assessment. American Sociological Review. 54: 400-423.

10. Heatherton TF, Kleck RE, Hebl MR, Hull JG. (2000) The social psychology of stigma. New York: Guilford Press.

11. Snowden LR, Pingitore D. (2002) Frequency and scope of mental health service delivery to African Americans in primary care. Mental Health Services Research. 4[3]:123-130.

12. Link BG, Cullen FT, Frank J, Wozniak JF. (1987) The social rejection of former mental patients: Understanding why labels matter. The American Journal of Sociology. 92[6]:1461-1500.

13. Vibha P, Saddichha S, Kumar R. (2008) Attitudes of ward attendants towards mental illness: Comparisons and predictors. International Journal of Social Psychiatry. 54 [5]: 469-478.

14. Dudley JR. (2000) Confronting stigma within the services system. Social Work. 45: 449-455.

15. Goffman E. (1984) Stigma: Notes on the Management of Spoiled Identity. Englewood Cliffs NJ: Prentice Hall; 1963.Jones EE, Farina A, Hastorf AH, Markus H, Miller DT, Scott RA. Social stigma: The psychology of marked relationships. New York: Freeman.

16. Feldman DB, Crandall CS. (2007) Dimensions of mental illness stigma: What about mental illness causes social rejection? Journal of Social and Clinical Psychology. 26[2]:137-154.

17. Corrigan PW, River LP, Lundin RK, Penn DL, Uphoff-Wasowski $\mathrm{K}$, Campion J, et al. (2001) Three strategies for changing attributions about severe mental illness. Schizophrenia Bulletin. 27[2]:187-195.

18. Corrigan PW, River LP, Lundin RK, Wasowski KU, Campion J, Mathisen J, et al. (2000) Stigmatizing attributions about mental illness. Journal of Community Psychology. 28 [1]: 91-102. 
19. Proctor EK. (2004) Research to inform mental health practice: Social work's contribution. Social Work Research. 28:195-197.

20. Rössler W. (2016) the stigma of mental disorders: A millennialong history of social exclusion and prejudices. EMBO reports. 17 [9]: 1250-1253.

21. Thornicroft G, Brohan E, Rose D, Sartorius N, Leese M, INDIGO Study Group. (2009) Global pattern of experienced and anticipated discrimination against people with schizophrenia: a cross-sectional survey. Lancet. 373: 408 - 415.

22. Nordt C, Rössler W, Lauber C. (2006) Attitudes of mental health professionals toward people with schizophrenia and major depression. Schizophr Bull. 32: 709 - 714.

23. Loch AA, Hengartner MP, Guarniero FB, Lawson FL, Wang YP, Gattaz WF, Rössler W. (2013) The more information, the more negative stigma towards schizophrenia: Brazilian general population and psychiatrists compared. Psychiatry Res. 205: 185 $-191$.

24. Rössler W, Salize HJ, van Os J, Riecher-Rössler A. (2005) Size of burden of schizophrenia and psychotic disorders. Eur Neuropsychopharmacol. 15: 399 - 409.

25. Ostman M, Kjellin L. (2002) Stigma by association: psychological factors in relatives of people with mental illness. $\mathrm{Br}$ J Psychiatry. 181: $494-498$.

26. Oexle N, Corrigan PW. (2018) Understanding mental illness stigma toward persons with multiple stigmatized conditions: implications of intersectionality theory. Psychiatric Serv. 69: 587589.

27. Corrigan P W. (2005) On the Stigma of Mental Illness: Practical Strategies for Research and Social Change. Washington, DC: American Psychological Association.

28. Schnyder N, Michel C, Panczak R, Ochsenbein S, Schimmelmann B G, Schultze-Lutter F. (2018) The interplay of etiological knowledge and mental illness stigma on healthcare utilization in the community: a structural equation model. Eur. Psychiatry. 51: $48-56$.

29. Angermeyer M C, Matschinger H. (2005) Causal beliefs and attitudes to people with schizophrenia. Trend analysis based on data from two population surveys in Germany. Br J. Psychiatry. 186: 331-334.

30. McGinty EE, Goldman HH, Pescosolido BA, and Barry CL. (2018) Communicating about mental illness and violence: balancing stigma and increased support for services. J. Health Polit. Policy Law. 43: 185-228.

31. Mannarini S, Boffo M. (2015) Anxiety, bulimia, drug and alcohol addiction, depression, and schizophrenia: what do you think about their etiology, dangerousness, social distance, and treatment? A latent class analysis approach.Soc. Psychiatry Psychiatr. Epidemiol. 50: 27-37.

32. Corrigan, P. W. [2000]. Mental health stigma as social attribution: implications for research methods and attitude change. Clin. Psychol. Sci. Prac. 7: 48-67.

33. Read J, Haslam N, Sayce L, Davies E. (2006) Prejudice and schizophrenia: a review of the "mental illness is an illness like any other" approach. Acta Psychiatr. Scand. 114: 303-318.
34. Schomerus G, Schwahn C, Holzinger A, Corrigan P W, Grabe H J, Carta M G, et al. (2012) Evolution of public attitudes about mental illness: a systematic review and metaanalysis. Acta Psychiatr. Scand. 125: 440-452.

35. Lefley HP. (1989) Family burden and family stigma in major mental illness. American Psychologist. 44[3]:556-560.

36. Thornicroft G, Brohan E, Kassam A, Lewis-Holmes E. (2008) Reducing stigma and discrimination: Candidate interventions. International Journal of Mental Health Systems. 2:3.

37. Britten N. (1998) Psychiatry, stigma, and resistance: Psychiatrists need to concentrate on understanding, not simply compliance. BMJ. 317: 963.

38. Pescosolido BA, Fettes DL, Martin JK, Monahan J, McLeod JD. (2007) Perceived dangerousness of children with mental health problems and support for coerced treatment. Psychiatric Services. 58[5]: 619-625.

39. Klin A, Lemish D. (2008) Mental disorders stigma in the media: Review of studies on production, content, and influences. Journal of Health Communication. 13[5]:434-449.

40. Corrigan PW. (2011) Best practices: Strategic stigma change [SSC]: Five principles for social marketing campaigns to reduce stigma. Psychiatric Services. 62 [8]: 824-826.

41. Parcesepe AM, Cabassa LJ. (2013) Public Stigma of Mental Illness in the United States: A Systematic Literature Review. Adm Policy Ment Health. 40[5]: 1-22.

42. Angermeyer MC, Holzinger A, Matschinger H. (2009) Mental health literacy and attitude towards people with mental illness: A trend analysis based on population surveys in eastern part of Germany. European Psychiatry. 24:225-232.

43. Liberman RP. (1988) Psychiatric Rehabilitation of Chronic Mental Patients. American Psychiatric Association Publishing; first edition, VA, USA.

44. World Health Organization. The World Health Report 2001. Mental Health: New Understanding, New Hope. Geneva: World Health Organization; 2001.

45. Jablensky A. (2017) Worldwide Burden of Schizophrenia. In: Sadock BJ, Sadock VA, Ruiz P, eds. Kaplan \& Sadock's Comprehensive Textbook of Psychiatry. 10th ed. Philadelphia: Wolters Kluwer. 3666-3697.

46. Hopper K, Harrison G, Janca A, Sartorius N, eds. (2007) Recovery From Schizophrenia. An International Perspective. A Report from the WHO Collaborative Project, The International Study of Schizophrenia. Oxford: Oxford University Press.

47. Fabrega H. (1991) Psychiatric stigma in non-Western societies. Compr Psychiatry. 32: 534-551.

48. $\mathrm{Ng} \mathrm{CH}$. (1996) The stigma of mental illness in Asian cultures. Aust N Zeal J Psychiatry. 31: 382-90.

49. Shahveysi N, Shoja Shafti S, Fadaie F, Dolatshahi B. (2007) Comparison of mental illness stigmatization in families of schizophrenic and major depressive disorder patients without psychotic features. Journal of Rehabilitation. 8[29]: 21-27.

50. Dols MW. (1987) Insanity and its treatment in Islamic society. Med History. 31:1-14.

51. Mannarini S, Rossi A. (2019) Assessing Mental Illness Stigma: A Complex Issue. Frontiers in Psychology; 9: 1-5. 